\title{
Extrinsic CPT Violation in Neutrino Oscillations
}

\author{
Tommy Ohlsson ${ }^{1}$
}

\begin{abstract}
Division of Mathematical Physics, Department of Physics, Royal Institute of Technology (KTH) Stockholm Center for Physics, Astronomy, and Biotechnology (SCFAB), Roslagstullsbacken 11, SE-10691 Stockholm, Sweden
\end{abstract}

\begin{abstract}
In this talk, we investigate extrinsic CPT violation in neutrino oscillations in matter with three flavors. Note that extrinsic CPT violation is different from intrinsic CPT violation. Extrinsic CPT violation is one way of quantifying matter effects, whereas intrinsic CPT violation would mean that the CPT invariance theorem is not valid. We present analytical formulas for the extrinsic CPT probability differences and discuss their implications for long-baseline experiments and neutrino factory setups.
\end{abstract}

Introduction. Recently, there have been several studies on CPT violation in order to incorporate the results of the LSND experiment [1], which require a third mass squared difference. However, this is not compatible with three neutrino flavors. Therefore, in most of the phenomenological studies on CPT violation, different mass squared differences and mixing parameters for neutrinos and antineutrinos are introduced by hand leading to four mass squared differences and eight mixing parameters. Thus, it is possible to include the results of the LSND experiment. Note that the results of the LSND experiment will be tested by the MiniBooNE experiment (September $2002 \rightarrow \sim 2005$ ) [2]. Furthermore, note that another possible description of the results of the LSND experiment are sterile neutrinos. However, sterile neutrinos have, in principle, been excluded by the SNO experiment [3]. Moreover, the first KamLAND data are consistent with the LMA solution [4], which means that there is no need for fundamental CPT violation.

Eccentric or extrinsic CPT violation? Let us denote the neutrino oscillation transition probabilities by $P_{\alpha \beta} \equiv P\left(v_{\alpha} \rightarrow v_{\beta}\right)$. Then, the CP, T, and CPT probability differences (pds) are defined as $\Delta P_{\alpha \beta}^{\mathrm{CP}} \equiv P_{\alpha \beta}-P_{\bar{\alpha} \bar{\beta}}, \Delta P_{\alpha \beta}^{\mathrm{T}} \equiv P_{\alpha \beta}-P_{\beta \alpha}$, and $\Delta P_{\alpha \beta}^{\mathrm{CPT}} \equiv P_{\alpha \beta}-P_{\bar{\beta} \bar{\alpha}}$.

Now, intrinsic (eccentric) CPT violation (or fundamental or genuine CPT violation) is due to violation of the CPT invariance theorem, whereas extrinsic CPT violation (or matter-induced or fake CPT violation) is due to presence of ordinary matter. Here, we will assume that the CPT invariance theorem is valid. This implies for the $\mathrm{CP}$ and $\mathrm{T}$ pds that the intrinsic and extrinsic effects are mixed, whereas for the CPT pds there are extrinsic effects only. Therefore, non-zero (extrinsic) CPT pds show matter effects, and thus, they are one way of quantifying such effects.

From conservation of probability, we obtain $\sum_{\alpha=e, \mu, \tau, \ldots} \Delta P_{\alpha \beta}^{\mathrm{CPT}}=\sum_{\beta=e, \mu, \tau, \ldots} \Delta P_{\alpha \beta}^{\mathrm{CPT}}=0$.

${ }^{1}$ In collaboration with: Magnus Jacobson. 
Note that not all of these equations are linearly independent. For three neutrino flavors, we have nine CPT pds for neutrinos. However, only four are linearly independent. Choosing, e.g., $\Delta P_{e e}^{\mathrm{CPT}}, \Delta P_{e \mu}^{\mathrm{CPT}}, \Delta P_{\mu e}^{\mathrm{CPT}}$, and $\Delta P_{\mu \mu}^{\mathrm{CPT}}$ as the known ones, the other five can be expressed in terms of these. Furthermore, we have $\Delta P_{\alpha \beta}^{\mathrm{CPT}}=-\Delta P_{\bar{\beta} \bar{\alpha}}^{\mathrm{CPT}}$, i.e., the CPT pds for antineutrinos do not give any further information.

The CPT probability differences. In vacuum, the CPT pds are $\Delta P_{\alpha \beta}^{\mathrm{CPT}}=0, \quad \alpha, \beta=$ $e, \mu, \tau$, whereas, in matter, they are given by $\Delta P_{\alpha \beta}^{\mathrm{CPT}}=\left|\left[S_{f}\left(t, t_{0}\right)\right]_{\beta \alpha}\right|^{2}-\left|\left[\bar{S}_{f}\left(t, t_{0}\right)\right]_{\alpha \beta}\right|^{2}$, where $S_{f} \equiv S_{f}\left(t, t_{0}\right)$ and $\bar{S}_{f} \equiv \bar{S}_{f}\left(t, t_{0}\right)$ are the evolution operators for neutrinos and antineutrinos, respectively. We have calculated $S_{f}$ and $\bar{S}_{f}$ explicitly using first order perturbation theory in the small leptonic mixing angle $\theta_{13}$. These explicit expressions for $S_{f}$ and $\bar{S}_{f}$ can be found in Ref. [5].

Two of the CPT pds (with an arbitrary matter density profile) are: $\Delta P_{e e}^{\mathrm{CPT}} \simeq|\bar{\beta}|^{2}-|\beta|^{2}$ and $\Delta P_{e \mu}^{C P T} \simeq c_{23}^{2}\left(|\beta|^{2}-|\bar{\beta}|^{2}\right)-2 c_{23} s_{23} \mathfrak{I}\left(\beta f C-\bar{\beta} \bar{f}^{*} \bar{A}^{*}\right)$, where $\beta$ and $\bar{\beta}$ describe a part of the two flavor neutrino evolution in the (1,2)-subsector, $f$ and $\bar{f}$ are some functions, and $\bar{A}$ and $C$ are complicated functions that can be found in Ref. [5].

In matter of constant density in the low-energy region $(V \lesssim \delta \ll \Delta)$, the CPT pds $\Delta P_{e e}^{\mathrm{CPT}}$ and $\Delta P_{\mu e}^{\mathrm{CPT}}$ are calculated to be [5]

$$
\begin{aligned}
\Delta P_{e e}^{\mathrm{CPT}} & \simeq 8 s_{12}^{2} c_{12}^{2} \cos 2 \theta_{12}\left(\delta L \cos \frac{\delta L}{2}-2 \sin \frac{\delta L}{2}\right) \sin \frac{\delta L}{2} \frac{V}{\delta}+\mathscr{O}\left((V / \delta)^{3}\right) \\
\Delta P_{\mu e}^{\mathrm{CPT}} & \simeq-8 s_{12}^{2} c_{12}^{2} c_{23}^{2} \cos 2 \theta_{12}\left(\delta L \cos \frac{\delta L}{2}-2 \sin \frac{\delta L}{2}\right) \sin \frac{\delta L}{2} \frac{V}{\delta} \\
& -16 s_{12} c_{12}^{3} s_{13} s_{23} c_{23} \cos \delta_{\mathrm{CP}} \cos 2 \theta_{12}\left(\delta L \cos \frac{\delta L}{2}-2 \sin \frac{\delta L}{2}\right) \sin \frac{\delta L}{2} \frac{V}{\delta} \\
& +16 s_{12} c_{12} s_{13} s_{23} c_{23} \sin \delta_{\mathrm{CP}}\left\{\cos 2 \theta_{12}[\delta L \cos \delta L-\cos \Delta L\right. \\
& \left.\left.\times\left(\delta L \cos \frac{\delta L}{2}-2 \sin \frac{\delta L}{2}\right)-\sin \delta L\right]+\delta L \sin \frac{\delta L}{2} \sin \Delta L\right\} \frac{V}{\delta}+\mathscr{O}\left((V / \delta)^{3}\right),
\end{aligned}
$$

where $\delta \equiv \frac{\Delta m_{21}^{2}}{2 E_{v}}, \Delta \equiv \frac{\Delta m_{31}^{2}}{2 E_{v}}, E_{v}$ is the neutrino energy, $L$ is the baseline length, and $V$ is the matter potential. Note that if one makes the replacement $\delta_{\mathrm{CP}} \rightarrow-\delta_{\mathrm{CP}}$, then $\Delta P_{e \mu}^{\mathrm{CPT}} \rightarrow$ $\Delta P_{\mu e}^{\mathrm{CPT}}$ and $\Delta P_{e \tau}^{\mathrm{CPT}} \rightarrow \Delta P_{\tau e}^{\mathrm{CPT}}$ and, in the case that $\delta_{\mathrm{CP}}=0$, one has $\Delta P_{e \mu}^{\mathrm{CPT}}=\Delta P_{\mu e}^{\mathrm{CPT}}$ and $\Delta P_{e \tau}^{\mathrm{CPT}}=\Delta P_{\tau e}^{\mathrm{CPT}}$. We observe also that in $\Delta P_{e e}^{\mathrm{CPT}}$ there are no $\delta_{\mathrm{CP}}$ terms, whereas in $\Delta P_{\mu e}^{\mathrm{CPT}}$ there are both $\sin \delta_{\mathrm{CP}}$ and $\cos \delta_{\mathrm{CP}}$ terms. Therefore, it would be possible to extract $\delta_{\mathrm{CP}}$ from $\Delta P_{\mu e}^{\mathrm{CPT}}$. Actually, for symmetric matter density profiles it can be shown that the $\Delta P_{\alpha \beta}^{\mathrm{CPT}}$, s are always odd functions with respect to the matter potential $V[6,5]$.

In the case of a step-function matter density profile in the low-energy region $\left(V_{1,2} \lesssim\right.$ $\delta \ll \Delta), \Delta P_{e e}^{\mathrm{CPT}}$ is found to be [5]

$$
\Delta P_{e e}^{\mathrm{CPT}} \simeq 8 s_{12}^{2} c_{12}^{2} \cos 2 \theta_{12}\left[\delta\left(L_{1} \frac{V_{1}}{\delta}+L_{2} \frac{V_{2}}{\delta}\right) \cos \frac{\delta\left(L_{1}+L_{2}\right)}{2}\right.
$$


TABLE 1. Extrinsic CPT pds for some past, present, and fututre long-baseline experiments.

\begin{tabular}{|c|c|c|c|c|c|}
\hline \multirow[t]{2}{*}{ Experiment } & \multicolumn{2}{|c|}{ CPT probability differences } & \multirow[t]{2}{*}{ Experiment } & \multicolumn{2}{|c|}{ CPT probability differences } \\
\hline & Quantities & Numerical value & & Quantities & Numerical value \\
\hline BNL NWG & $\Delta P_{\mu e}^{\mathrm{CPT}}$ & 0.010 & KamLAND & $\Delta P_{e e}^{\mathrm{CPT}}$ & -0.033 \\
\hline BNL NWG & $\Delta P_{\mu e}^{\mathrm{CPT}}$ & 0.032 & LSND & $\Delta P_{\mu e}^{e e}$ & $4.8 \cdot 10^{-15}$ \\
\hline BooNE & $\Delta P_{\mu e}^{\mathrm{CPT}}$ & $6.6 \cdot 10^{-13}$ & MINOS & $\Delta P_{\mu e}^{\mathrm{CPT}}$ & $1.9 \cdot 10^{-4}$ \\
\hline MiniBooNE & $\Delta P_{\mu e}^{\mathrm{CPT}}$ & $4.1 \cdot 10^{-14}$ & & $\Delta P_{u \mu}^{\mathrm{CPT}}$ & $-1.1 \cdot 10^{-5}$ \\
\hline CHOOZ & $\Delta P_{e e}^{\mathrm{CPT}}$ & $-3.6 \cdot 10^{-5}$ & NuMI I & $\Delta P_{\mu e}^{\mathrm{CPT}}$ & 0.026 \\
\hline ICARUS & $\Delta P_{\mu e}^{\mathrm{CP}}$ & $4.0 \cdot 10^{-5}$ & NuMI II & $\Delta P_{\mu e}^{\mathrm{CPT}}$ & $2.6 \cdot 10^{-3}$ \\
\hline & $\Delta P_{\mu \tau}^{\mathrm{CPT}}$ & $-3.8 \cdot 10^{-5}$ & NuTeV & $\Delta P_{\mu e}^{\mu \mathrm{CPT}}$ & $1.6 \cdot 10^{-18}$ \\
\hline JHF-Kamioka & $\Delta P_{\mu e}^{\mathrm{CPT}}$ & $3.8 \cdot 10^{-3}$ & NuTeV & $\Delta P_{\mu e}^{\mathrm{CPT}}$ & $8.2 \cdot 10^{-20}$ \\
\hline & $\Delta P_{\mu \mu}^{\mathrm{CPT}}$ & $-1.3 \cdot 10^{-4}$ & OPERA & $\Delta P_{\mu \tau}^{\mathrm{CPT}}$ & $-3.8 \cdot 10^{-5}$ \\
\hline $\mathrm{K} 2 \mathrm{~K}$ & $\Delta P_{\mu e}^{\mathrm{CPT}}$ & $1.0 \cdot 10^{-3}$ & Palo Verde & $\Delta P_{e e}^{\mathrm{CPT}}$ & $-1.2 \cdot 10^{-5}$ \\
\hline & $\Delta P_{\mu \mu}^{\mathrm{CPT}}$ & $-5.3 \cdot 10^{-5}$ & Palo Verde & $\Delta P_{e e}^{\mathrm{CPT}}$ & $-2.2 \cdot 10^{-5}$ \\
\hline
\end{tabular}

$$
\left.-2\left(\frac{V_{1}}{\delta} \sin \frac{\delta L_{1}}{2} \cos \frac{\delta L_{2}}{2}+\frac{V_{2}}{\delta} \sin \frac{\delta L_{2}}{2} \cos \frac{\delta L_{1}}{2}\right)\right] \sin \frac{\delta\left(L_{1}+L_{2}\right)}{2} .
$$

Note that this formula is completely symmetric with respect to interchange of layers 1 and 2 and, in the limit $V_{1,2} \rightarrow V$ and $L_{1,2} \rightarrow L / 2$, one has $\Delta P_{e e}^{C P T}$ (step-function) $\rightarrow$ $\Delta P_{e e}^{\mathrm{CPT}}$ (constant).

Similarly, in the case of the T probability difference in the low-energy region $(\delta=$ $\left.\Delta m_{21}^{2} /\left(2 E_{v}\right) \gtrsim V_{1,2}\right)$, one finds [7]

$$
\begin{aligned}
\Delta P_{\alpha \beta}^{\mathrm{T}} \simeq \cos \delta_{\mathrm{CP}} \cdot 8 \underbrace{8 s_{12} c_{12} s_{13} s_{23} c_{23} \frac{\sin \left(2 \theta_{1}-2 \theta_{2}\right)}{\sin 2 \theta_{12}}}_{J_{\text {eff }}}\left\{s_{1} s_{2}\left[Y-\cos \left(\Delta_{1} L_{1}+\Delta_{2} L_{2}\right)\right]\right\} \\
+\sin \delta_{\mathrm{CP}} \cdot 4 s_{13} s_{23} c_{23} X_{1}\left[Y-\cos \left(\Delta_{1} L_{1}+\Delta_{2} L_{2}\right)\right],
\end{aligned}
$$

where $J_{\text {eff }}$ is an effective Jarlskog invariant. (See Ref. [7] for the definitions of the different quantities in this formula.) Here the $\cos \delta_{C P}$ term is due to matter-induced $\mathrm{T}$ violation, whereas the usual $\sin \delta_{C P}$ term is due to fundamental T violation.

Numerical calculations and implications. Using the present best-fit values of the fundamental neutrino parameters, $\Delta m_{21}^{2} \simeq 7.1 \cdot 10^{-5} \mathrm{eV}^{2},\left|\Delta m_{31}^{2}\right| \simeq 2.5 \cdot 10^{-3} \mathrm{eV}^{2}$, $\theta_{12} \simeq 34^{\circ}, \theta_{23} \simeq 45^{\circ}$, and, in addition, choosing a normal mass hierarchy spectrum $\left[\operatorname{sgn}\left(\Delta m_{31}^{2}\right)=1\right], \theta_{13}=9.2^{\circ}$, and $\delta_{\mathrm{CP}}=0$, we have calculated the CPT pds for some past, present, and future long-baseline experiments. The results are presented in Table 1. Note that $\Delta P_{e e}^{\mathrm{CPT}}$ for the KamLAND experiment is $\left|\Delta P_{e e}^{\mathrm{CPT}}\right| \sim 3 \%$, which means that extrinsic CPT violation is non-negligible for this experiment. The problem is just how one should obtain $P_{e e}$ for the same neutrino energy and baseline length. Furthermore, we have calculated $\Delta P_{\mu e}^{C P T}$ for two neutrino factory setups, using the following parameter values: $\rho=\rho_{\text {mantle }} \simeq 4.5 \mathrm{~g} / \mathrm{cm}^{3}, E_{v}=50 \mathrm{GeV}, L \in\{3000,7000\} \mathrm{km}$. Then, $L=3000 \mathrm{~km}$ leads to $\Delta P_{\mu e}^{\mathrm{CPT}} \simeq 3.0 \cdot 10^{-5}$, whereas $L=7000 \mathrm{~km}$ leads to $\Delta P_{\mu e}^{\mathrm{CPT}} \simeq 1.8 \cdot 10^{-5}$. Therefore, extrinsic $\mathrm{CPT}$ violation is practically negligible for a future neutrino factory. In Fig. $1, \Delta P_{e e}^{C P T}$ and $\Delta P_{\mu e}^{C P T}$ are shown plotted as functions of 

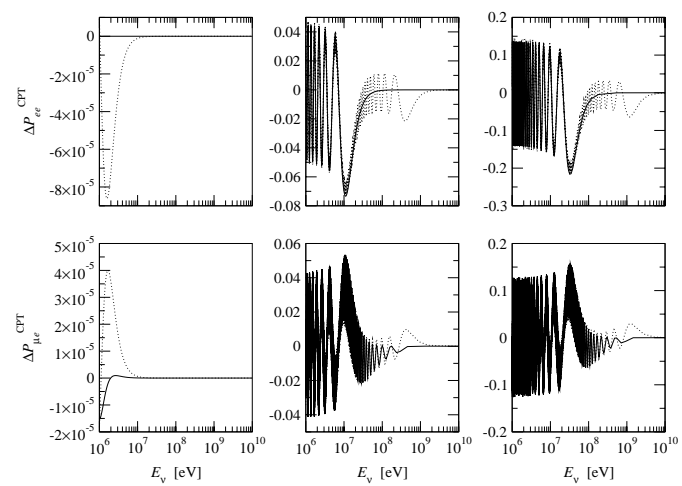

FIGURE 1. The CPT pds $\Delta P_{e e}^{\mathrm{CPT}}$ and $\Delta P_{\mu e}^{\mathrm{CPT}}$ as functions of the neutrino energy $E_{v}$ for baseline lengths $L \in\{1,250,750\} \mathrm{km}$. The solid curves show analytical results, whereas the dotted curves show numerical results. The fast oscillations present in the numerical results are averaged out in the analytical calculations.

$E_{v}$ for $L \in\{1,250,750\} \mathrm{km}$. We note that an increasing $L$ implies an increasing values of the $\Delta P_{\alpha \beta}^{C P T}$ 's and when $E_{v} \rightarrow \infty$ we observe that $\Delta P_{\alpha \beta}^{C P T} \rightarrow 0$.

Summary \& Conclusions. In conclusion, we have studied extrinsic CPT violation in three flavor neutrino oscillations assuming the CPT invariance theorem. In general, the (extrinsic) CPT pds for an arbitrary matter density profile have been derived. In particular, first order perturbation theory formulas for constant and step-function matter density profiles have been calculated as well as low-energy approximations. Furthermore, implications for accelerator and reactor long-baseline experiments as well as neutrino factory setups have been presented. For certain experiments the CPT pds can be as large as $\left|\Delta P_{\alpha \beta}^{\mathrm{CPT}}\right| \sim 5 \%$. In general, the CPT pds increase with increasing baseline length and decrease with increasing neutrino energy.

Acknowledgments. I would like to thank S.M. Bilenky, M. Jacobson, R. Johansson, M. Lindner, H. Minakata, G. Seidl, H. Snellman, and W. Winter for useful discussions and comments. This work was supported by the Swedish Research Council (Vetenskapsrådet), Contract No. 621-2001-1611, 621-2002-3577, the Magnus Bergvall Foundation (Magn. Bergvalls Stiftelse), and the Wenner-Gren Foundations.

\section{REFERENCES}

1. Athanassopoulos, C., et al., Phys. Rev. Lett., 77, 3082-3085 (1996); 81, 1774-1777 (1998); Aguilar, A., et al., Phys. Rev., D64, 112007 (2001).

2. http://www-boone.fnal.gov/.

3. Bahcall, J. N., Gonzalez-Garcia, M. C., and Peña-Garay, C., J. High Energy Phys., 07, 054 (2002).

4. Bahcall, J. N., Gonzalez-Garcia, M. C., and Peña-Garay, C., J. High Energy Phys., 02, 009 (2003).

5. Jacobson, M., and Ohlsson, T., hep-ph/0305064.

6. Minakata, H. (2003), private communication.

7. Akhmedov, E. K., Huber, P., Lindner, M., and Ohlsson, T., Nucl. Phys., B608, 394-422 (2001). 